\title{
Bellman-Gronwall Inequality Approach to Oscillator Design for the Specific Bilinear Systems
}

\author{
Yeong-Jeu Sun*
}

Department of Electrical Engineering, I-Shou University, Taiwan, R.O.C.

\begin{abstract}
In this paper, the existence of limit cycles for the specific bilinear systems is explored. Based on the BellmanGronwall inequality approach, not only the exponentially stable limit cycles phenomenon of such systems can be certified but also the oscillation behaviors of such systems can be correctly predicted. Finally, a numerical example is provided to illustrate the feasibility and effectiveness of the obtained result.
\end{abstract}

\section{INTRODUCTION}

Nonlinear system can offer oscillations with fixed amplitude and fixed frequency. These oscillations are named limit cycles, e.g., an RLC electrical circuit with a nonlinear resistor and Van der Pol equation. Limit cycles are singular phenomenon of nonlinear systems and have been a main interest of the researchers over the years; see, for example, [1-9], and the references therein. Prediction of limit cycles is very important, because limit cycles can appear in any kind of physical system. Ordinarily, a limit cycle can be desirable. This is the case of limit cycles in the electronic oscillators utilized in laboratories. There are at least two approaches to explore the phenomenon of limit cycles, namely describing function method and Poincare-Bendixson theorem. The disadvantages of the describing function approach are related to its approximate nature, and include the possibility of incorrect predictions. Besides, the Poincare-Bendixson theorem only offers a necessary condition to guarantee the existence of limit cycles. Consequently, if any one of the conditions of the Poincare-Bendixson theorem is not satisfied for some system, it can be guaranteed that there exists no limit cycles in such system. Conversely, even the conditions of the Poincare-Bendixson theorem are satisfied for some system, the existence of limit cycles cannot be guaranteed for such system [9].

In this paper, based on the Bellman-Gronwall inequality approach, the exponentially stable limit cycles for the specific bilinear control systems can be guaranteed. Furthermore, an estimate of the guaranteed convergence rate is also derived for such systems.

\section{PROBLEM FORMULATION AND MAIN RESULT}

In this paper, we consider the following bilinear control systems [10]:

$$
\begin{aligned}
& \delta(t)=A z(t)+r u(t) z(t), \forall t \geq t_{0} \geq 0, \\
& z\left(t_{0}\right)=\left[\begin{array}{l}
z_{10} \\
z_{20}
\end{array}\right],
\end{aligned}
$$

*Address correspondence to this author at the Department of Electrical Engineering, I-Shou University, Taiwan, R.O.C.;

E-mail: yjsun@isu.edu.tw where

$$
\begin{gathered}
z=\left[\begin{array}{l}
z_{1} \\
z_{2}
\end{array}\right] \in \Re^{2}, \quad u \in \Re, \\
A:=\left[\begin{array}{cc}
-a w+d & -w\left(1+a^{2}\right) \\
w & a w+d
\end{array}\right],
\end{gathered}
$$

with $r \neq 0, a, d \in \Re, w \neq 0$, and $z\left(t_{0}\right) \neq 0$. Specially, the feedback control law is selected as follows

$$
u(t)=-\frac{1}{r}\left[\left(z_{1}(t)+a z_{2}(t)\right)^{2}+z_{2}^{2}-k^{2}+d\right],
$$

with $k>0$. Thus the closed-loop systems are deduced as

$$
\begin{gathered}
\left\{\begin{array}{l}
\&=-a w z_{1}-w\left(1-a^{2}\right) z_{2}-z_{1}\left[\left(z_{1}+a z_{2}\right)^{2}+z_{2}^{2}-k^{2}\right], \\
\&=w z_{1}+a w z_{2}-z_{2}\left[\left(z_{1}+a z_{2}\right)^{2}+z_{2}^{2}-k^{2}\right], \quad \forall t \geq t_{0} .
\end{array}\right. \\
x(t):=\left[\begin{array}{c}
x_{1}(t) \\
x_{2}(t)
\end{array}\right]=\left[\begin{array}{c}
z_{1}(t)+a z_{2}(t) \\
z_{2}(t)
\end{array}\right], \quad \forall t \geq t_{0},
\end{gathered}
$$

it can be obtained that

$$
\left\{\begin{array}{l}
\&(t)=-w x_{2}(t)-x_{1}(t)\left[x_{1}^{2}(t)+x_{2}^{2}(t)-k^{2}\right], \\
\&(t)=w x_{1}(t)-x_{2}(t)\left[x_{1}^{2}(t)+x_{2}^{2}(t)-k^{2}\right], \quad \forall t \geq t_{0} .
\end{array}\right.
$$

Obviously, $x=0$ is an equivalent point of system (3), i.e., the solution of system (3) is given by $x(t)=0$ if $x\left(t_{0}\right)=0$ (or equivalently $\left.z\left(t_{0}\right)=0\right)$. To avoid the trivial case of $x\left(t_{0}\right)=0$, in the following, we only consider the system (1) under the case of $x\left(t_{0}\right) \neq 0$.

\section{DEFINITION [9]}

Consider the system (1). The closed and bounded manifold $\bar{s}(z)=0$, in the $z_{1}-z_{2}$ plane, is said to be an exponentially stable limit cycle if there exist two positive numbers $\alpha$ and $\beta$ such that the manifold of $\bar{s}(z)=0$ along the trajectories of system (1) satisfies the following inequality 


$$
|\bar{s}(z(t))| \leq \beta \cdot \exp \left[-\alpha\left(t-t_{0}\right)\right] \forall t \geq t_{0} .
$$

In this case, the positive number $\alpha$ is called the guaranteed convergence rate.

Now, we present the main result for the existence of limit cycles of system (1) as follows.

\section{Theorem 1.}

For the feedback bilinear systems (1), all of phase trajectories tend to the exponentially stable limit cycle $\bar{s}(z)=\left(z_{1}+a z_{2}\right)^{2}+z_{2}^{2}-k^{2}=0$ in the $z_{1}-z_{2}$ plane, with the guaranteed convergence rate $2\left(z_{10}+a z_{20}\right)^{2}+2 z_{20}^{2}$. Furthermore, the states $z_{1}(t)$ and $z_{2}(t)$ exponentially track, respectively, the trajectories

and

$$
k \cos \left[w\left(t-t_{0}\right)+\theta_{0}\right]+a k \sin \left[w\left(t-t_{0}\right)+\theta_{0}\right]
$$

$$
\begin{gathered}
k \sin \left[w\left(t-t_{0}\right)+\theta_{0}\right], \\
\theta_{0}=\tan ^{-1}\left(\frac{z_{20}}{z_{10}+a z_{20}}\right),
\end{gathered}
$$

in the time domain, with the guaranteed convergence rate $\left(z_{10}+a z_{20}\right)^{2}+z_{20}^{2}$.

Proof. Define a smooth manifold $s(x)=0$ and a continuous function

with

$$
\theta(x):=\tan ^{-1}\left[\frac{x_{2}}{x_{1}}\right]
$$

$$
s(x):=x^{T} x-k^{2}=\left(z_{1}+a z_{2}\right)^{2}+z_{2}^{2}-k^{2}=s(z) .
$$

Then the time derivatives of $s(x)$ and $\theta(x)$ along the trajectories of system (3) is given by

$$
\begin{aligned}
\frac{d s(x(t))}{d t} & =2 x_{1} \cdot \alpha+2 x_{2} \alpha \\
& =-2\left(x_{1}^{2}+x_{2}^{2}\right)\left(x_{1}^{2}+x_{2}^{2}-k^{2}\right) ; \\
\frac{d \theta(x(t))}{d t} & =\frac{\delta x_{1}-\delta x_{2}}{x_{1}^{2}+x_{2}^{2}}=w,
\end{aligned}
$$

which imply

$$
\theta(x(t))=w\left(t-t_{0}\right)+\theta_{0} \text {. }
$$

In the following, there are three cases to discuss the trajectories of the feedback control system of (3).

Case 1: $x_{1}^{2}\left(t_{0}\right)+x_{2}^{2}\left(t_{0}\right)=k^{2}$

$\frac{d s(x(t))}{d t}=0$, which implies

In this case, by (4) and (5), it can be obtained that

$$
x_{1}^{2}(t)+x_{2}^{2}(t)=k^{2}, \forall t \geq t_{0} .
$$

Hence we conclude that

$$
\begin{gathered}
x_{1}(t)=k \cos \left[w\left(t-t_{0}\right)+\theta_{0}\right] \quad \forall t \geq t_{0} ; \\
x_{2}(t)=k \sin \left[w\left(t-t_{0}\right)+\theta_{0}\right] \quad \forall t \geq t_{0} ; \\
s(x(t))=0, \quad \forall t \geq t_{0},
\end{gathered}
$$

in view of (5) and (6). Thus, from (2), it can be shown that

$$
\begin{gathered}
z_{1}(t)=k \cos \left[w\left(t-t_{0}\right)+\theta_{0}\right]+a k \sin \left[w\left(t-t_{0}\right)+\theta_{0}\right] \forall t \geq t_{0} \\
z_{2}(t)=k \sin \left[w\left(t-t_{0}\right)+\theta_{0}\right] \quad \forall t \geq t_{0} \\
\bar{s}(z(t))=0, \quad \forall t \geq t_{0} .
\end{gathered}
$$

Case 2: $x_{1}^{2}\left(t_{0}\right)+x_{2}^{2}\left(t_{0}\right)>k^{2}$

In this case, by (4), it can be obtained that $s(x(t))$ is a strictly decreasing function of $t$ with $s(x(t)) \geq 0, \forall t \geq t_{0}$, and

$$
\frac{d s(x(t))}{d t} \leq-2 k^{2} s(x(t)), \forall t \geq t_{0} .
$$

Applying the Bellman-Gronwall inequality with above differential inequality, one has

$$
0 \leq s(x(t)) \leq s\left(x\left(t_{0}\right)\right) \cdot \exp \left[-2 k^{2}\left(t-t_{0}\right)\right], \quad \forall t \geq t_{0},
$$

which implies

$$
\begin{aligned}
\mid s & (x(t)) \mid \leq s\left(x\left(t_{0}\right)\right) \cdot \exp \left[-2 k^{2}\left(t-t_{0}\right)\right], \quad \forall t \geq t_{0} \\
k & \leq \sqrt{x_{1}^{2}(t)+x_{2}^{2}(t)} \\
& \leq \sqrt{k^{2}+s\left(x\left(t_{0}\right)\right) \cdot \exp \left[-2 k^{2}\left(t-t_{0}\right)\right]} \\
& \leq k+\sqrt{s\left(x\left(t_{0}\right)\right)} \cdot \exp \left[-k^{2}\left(t-t_{0}\right)\right], \quad \forall t \geq t_{0} .
\end{aligned}
$$

Consequently, by (5) and (7), we conclude that

$$
\begin{aligned}
& \left|x_{1}(t)-k \cos \left[w\left(t-t_{0}\right)+\theta_{0}\right]\right| \\
& \leq \sqrt{s\left(x\left(t_{0}\right)\right)} \cdot \exp \left[-k^{2}\left(t-t_{0}\right)\right], \quad \forall t \geq t_{0} ; \\
& \left|x_{2}(t)-k \sin \left[w\left(t-t_{0}\right)+\theta_{0}\right]\right| \\
& \leq \sqrt{s\left(x\left(t_{0}\right)\right)} \cdot \exp \left[-k^{2}\left(t-t_{0}\right)\right], \quad \forall t \geq t_{0} ; \\
& |s(x(t))| \leq s\left(x\left(t_{0}\right)\right) \cdot \exp \left[-2 k^{2}\left(t-t_{0}\right)\right], \quad \forall t \geq t_{0} .
\end{aligned}
$$

Thus, from (2), it can be shown that

$$
\begin{aligned}
& \left|z_{1}(t)-k \cos \left[w\left(t-t_{0}\right)+\theta_{0}\right]-a k \sin \left[w\left(t-t_{0}\right)+\theta_{0}\right]\right| \\
= & \mid x_{1}(t)+a x_{2}(t)-k \cos \left[w\left(t-t_{0}\right)+\theta_{0}\right] \\
& -a k \sin \left[w\left(t-t_{0}\right)+\theta_{0}\right] \mid \\
\leq & \left|x_{1}(t)-k \cos \left[w\left(t-t_{0}\right)+\theta_{0}\right]\right| \\
& +|a| \cdot\left|x_{2}(t)-k \sin \left[w\left(t-t_{0}\right)+\theta_{0}\right]\right| \\
\leq & (1+|a|) \cdot \sqrt{s\left(x\left(t_{0}\right)\right)} \cdot \exp \left[-k^{2}\left(t-t_{0}\right)\right], \quad \forall t \geq t_{0} ; \\
& \left|z_{2}(t)-k \sin \left[w\left(t-t_{0}\right)+\theta_{0}\right]\right| \quad \forall t \geq t_{0} ; \\
\leq & \sqrt{s\left(x\left(t_{0}\right)\right)} \cdot \exp \left[-k^{2}\left(t-t_{0}\right)\right], \quad \forall
\end{aligned}
$$




$$
|\bar{s}(z(t))| \leq s\left(x\left(t_{0}\right)\right) \cdot \exp \left[-2 k^{2}\left(t-t_{0}\right)\right], \quad \forall t \geq t_{0} .
$$

Case 3: $x_{1}^{2}\left(t_{0}\right)+x_{2}^{2}\left(t_{0}\right)=k_{0}^{2}<k^{2}, k_{0} \neq 0$

In this case, by (4), it can be obtained that $-s(x(t))$ is a strictly decreasing function of $t$ with $-s(x(t)) \geq 0, \forall t \geq t_{0}$, and

$$
\frac{d[-s(x(t))]}{d t} \leq-2 k_{0}^{2}[-s(x(t))] \forall t \geq t_{0} .
$$

Applying the Bellman-Gronwall inequality with above differential inequality, one has

$$
\begin{aligned}
0 & \leq-s(x(t)) \\
& \leq-s\left(x\left(t_{0}\right)\right) \cdot \exp \left[-2 k_{0}^{2}\left(t-t_{0}\right)\right], \quad \forall t \geq t_{0},
\end{aligned}
$$

which implies

$$
\begin{aligned}
k^{2}+s\left(x\left(t_{0}\right)\right) \cdot \exp \left[2 k_{0}^{2}\left(t-t_{0}\right)\right] & \leq x_{1}^{2}(t)+x_{2}^{2}(t) \\
& \leq k^{2}, \forall t \geq t_{0} ; \\
k-\sqrt{-s\left(x\left(t_{0}\right)\right)} \cdot \exp \left[k_{0}^{2}\left(t-t_{0}\right)\right] & \leq \sqrt{x_{1}^{2}(t)+x_{2}^{2}(t)} \\
\leq & k, \forall t \geq t_{0} .
\end{aligned}
$$

Consequently, by (5) and (8), we conclude that

$$
\begin{aligned}
& \left|x_{1}(t)-k \cos \left[w\left(t-t_{0}\right)+\theta_{0}\right]\right| \\
& \leq \sqrt{-s\left(x\left(t_{0}\right)\right)} \cdot \exp \left[-k_{0}^{2}\left(t-t_{0}\right)\right] ; \\
& \left|x_{2}(t)-k \sin \left[w\left(t-t_{0}\right)+\theta_{0}\right]\right| \\
& \leq \sqrt{-s\left(x\left(t_{0}\right)\right)} \cdot \exp \left[-k_{0}^{2}\left(t-t_{0}\right)\right] .
\end{aligned}
$$

From (2), it results that

$$
\begin{aligned}
&\left|z_{1}(t)-k \cos \left[w\left(t-t_{0}\right)+\theta_{0}\right]-a k \sin \left[w\left(t-t_{0}\right)+\theta_{0}\right]\right| \\
&= \mid x_{1}(t)+a x_{2}(t)-k \cos \left[w\left(t-t_{0}\right)+\theta_{0}\right] \\
&-a k \sin \left[w\left(t-t_{0}\right)+\theta_{0}\right] \mid \\
& \leq\left|x_{1}(t)-k \cos \left[w\left(t-t_{0}\right)+\theta_{0}\right]\right| \\
&+|a| \cdot\left|x_{2}(t)-k \sin \cos \left[w\left(t-t_{0}\right)+\theta_{0}\right]\right| \\
& \leq(1+|a|) \cdot \sqrt{-s\left(x\left(t_{0}\right)\right)} \cdot \exp \left[-k^{2}\left(t-t_{0}\right)\right], \quad \forall t \geq t_{0} ; \\
&\left|z_{2}(t)-k \sin \left[\left(w t-t_{0}\right)+\theta_{0}\right]\right| \\
& \leq \sqrt{-s\left(x\left(t_{0}\right)\right)} \cdot \exp \left[-k_{0}^{2}\left(t-t_{0}\right)\right] .
\end{aligned}
$$

This completes our proof.

\section{Remark 1.}

Obviously, by Theorem 1, the state of $x_{1}(t)$ can be represented as nonlinear oscillator with the amplitude $k \sqrt{1+a^{2}}$ and the frequency $\mathrm{w} \mathrm{rad} / \mathrm{sec}$. The state of $x_{2}(t)$ can be also represented as nonlinear oscillator with the amplitude $k$ and the frequency $w \mathrm{rad} / \mathrm{sec}$. Such oscillations are generally independent of the initial condition and limit cycles of such oscillation are not influenced by parameter variation.

\section{ILLUSTRATIVE EXAMPLE}

Consider the bilinear control system of (1) with

$$
a=1, w=r=2, d=3, t_{0}=0,
$$

and $z(0)=\left[\begin{array}{ll}4 & 4\end{array}\right]^{T}$. By Theorem 1 with $k=2$ and the control law

$$
u(t)=-\frac{1}{2}\left[z_{1}^{2}(t)+2 z_{2}^{2}(t)+2 z_{1}(t) z_{2}(t)-1\right],
$$

we conclude that the exponentially stable limit cycle is given by $\bar{s}(z)=z_{1}^{2}+2 x_{2}^{2}+2 z_{1} z_{2}-4=0$ in the $z_{1}-z_{2}$ plane, with the guaranteed convergence rate $\alpha=160$. Furthermore, the states $z_{1}(t)$ and $z_{2}(t)$ exponentially track, respectively, the trajectories $2 \cos (2 t+0.46)+2 \sin (2 t+0.46)$ and $2 \sin (2 t+0.46)$, in the time domain, with the guaranteed convergence rate $\left(z_{10}+a z_{20}\right)^{2}+z_{20}^{2}=80$. Some state trajectories of the feedback-controlled system are depicted in Fig. (1) Fig. (3).

$z_{1}(t)$

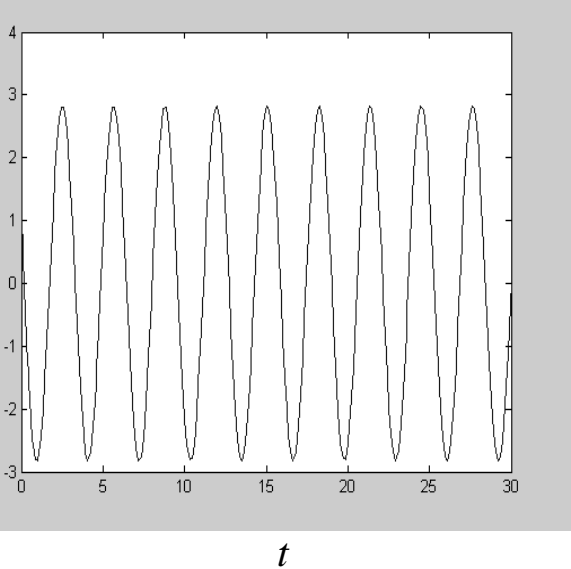

Fig. (1). $z_{1}(t)$ of the feedback-controlled system.

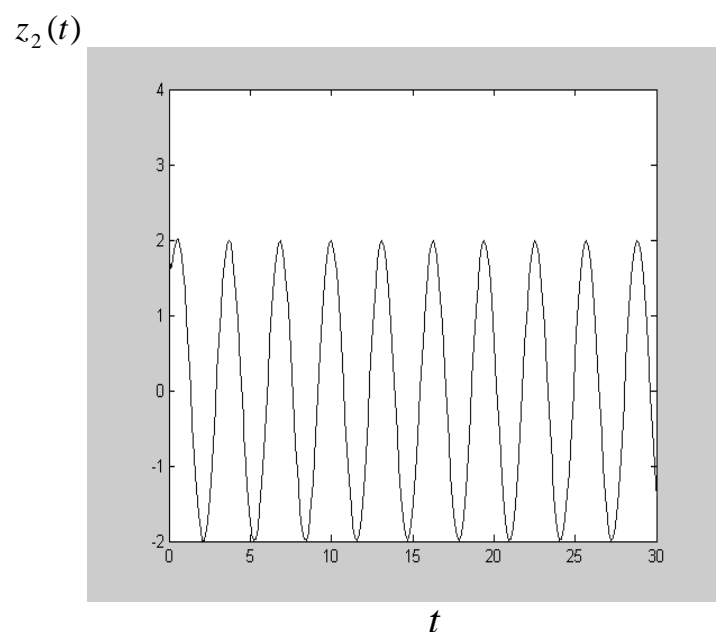

Fig. (2). $z_{2}(t)$ of the feedback-controlled system. 


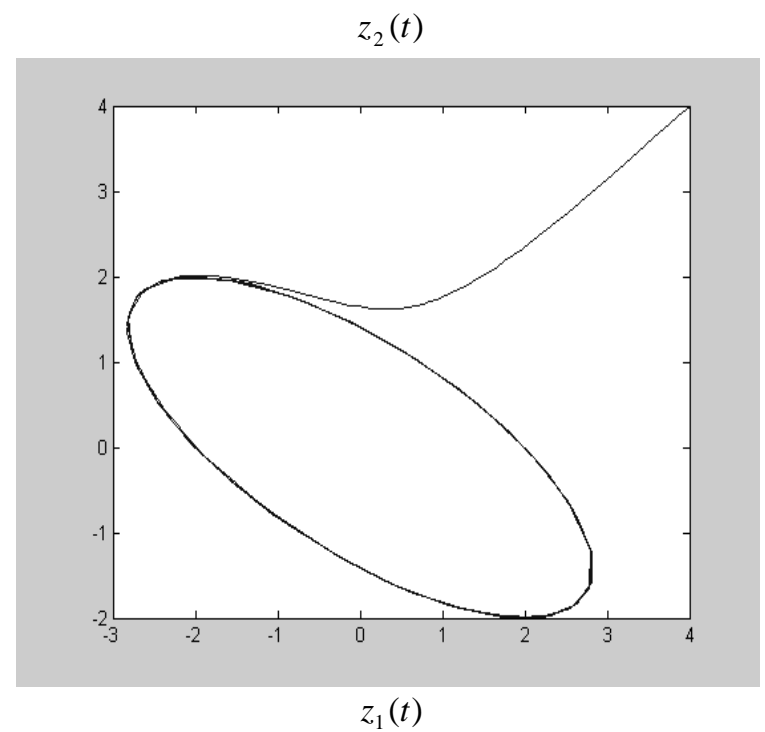

Fig. (3). $z_{1}(t)$ and $z_{2}(t)$ of the feedback-controlled.

\section{CONCLUSIONS}

In this paper, the existence of limit cycles for the specific bilinear control systems has been explored. Based on the Bellman-Gronwall inequality approach, not only the exponentially stable limit cycles phenomenon of such systems has been certified but also the oscillation behaviors of such systems has been correctly predicted. A numerical example has also been given to illustrate the feasibility and effectiveness of the obtained result. It is interesting to of the obtained result. It is interesting to consider the oscillator design problem with more general systems.

\section{ACKNOWLEDGMENT}

The author thanks the National Science Council of Republic of China for supporting this work under grant NSC96-2221-E-214-035.

\section{REFERENCES}

[1] P. Acosta, A. Polyakov, L. Fridman, and V. Strygin, “Estimation of amplitude of oscillations in sliding mode systems caused by time delay". Asian J. Control, vol. 6, pp. 517-513, 2004.

[2] D.P. Atherton, "Non-linear Control Engineering"' London, Van Nostrand Rienhold, 1975.

[3] Z. Cheng, Y. Li, and J. Cao. "Dynamical behaviors of a partialdependent predator-prey system,' Chaos, Solitons \& Fractals, vol 28, pp. 67-75, 2006.

[4] C.C. Hwang, J.Y. Hsieh and R.S. Lin, "A linear continuous feedback control of Chua's circuit', Chaos, Solitons \& Fractals, vol. 8, pp. 1507-15, 1997.

[5] H. Ohta, and Y. Ueda, "Blue sky bifurcations caused by unstable limit cycle leading to voltage collapse in an electric power system', Chaos, Solitons \& Fractals, vol. 14, pp. 127-137, 2002.

[6] Z.J. Palmor, Y. Halevi, and T. Efrati, "A general and exact method for determining limit cycles in decentralized relay systems", Automatica, vol. 31, pp. 1333-1339, 1995.

[7] J.I. Ramos, "Piecewise-linearized methods for oscillators with limit cycles. Chaos,' Solitons \& Fractals, vol. 27, pp. 1229-1238, 2006.

[8] Y.J. Sun, "Prediction of limit cycles in feedback bilinear systems: Lyapunov-like approach', In: Proceeding of 2002 Natioanl Symposium on Automatic Control; 2002. pp. 801-5.

[9] Y.J. Sun, "Limit cycles design for a class of bilinear control systems', Chaos, Solitons \& Fractals, vol. 33, pp. 156-162, 2007.

[10] P.R. Mohler, Bilinear Control Process. Academic Press, New York, 1973. 